\title{
PARAMETER ESTIMATION IN A STRUCTURED ALGAL COAGULATION-FRAGMENTATION MODEL
}

\author{
AZMY S. ACKLEH ${ }^{\dagger}$
}

\begin{abstract}
Coagulation of phytoplankton is a fundamental mechanism for vertical flux of carbon in the ocean. This process is dependent on parameters that are not available from experimental data, such as the encounter rate of particles, the contact efficiency of unlike particles and the probability of sticking upon contact. Fragmentation, the breakup of large particles into two smaller ones has been observed in the ocean, but very little modeling effort for incorporating this process in the dynamics of phytoplankton has been attempted. In this paper we incorporate fragmentation process into a nonlinear hyperbolic equation that describes the evolution of a size structured algal population with the aggregation model. We examine through numerical simulation the effect of fragmentation on the dynamics of phytoplankton. We present convergence theory for estimating parameters in this model using nonlinear least squares fit. The least square method is then tested numerically in ideal cases where the data is the model output with some added noise.
\end{abstract}

\section{Introduction}

Phytoplankton populations are large contributors to the primary production in the ocean. Due to the importance of the increase of $\mathrm{CO}_{2}$ in the atmosphere

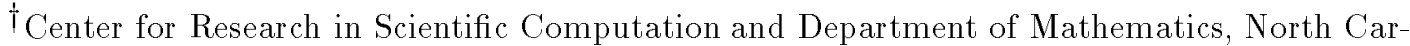
olina State University, Raleigh, NC 27695-8205. Research supported by in part by AFOSR grant F49620-93-1-0198
} 
and its effect on the global climate change, it is important to understand the role of phytoplankton in the process of transferring carbon to the deep sea by sedimentation and degradation of these cells.

The importance of coagulation process in oceanic plankton community [5, 6, 23] has been studied through many mathematical models [17, 18, 24, 14, 12, 22, 15, 3]. A common conclusion of these models is that aggregation enhances the sedimentation of phytoplankton cells to the bottom of the ocean. The fragmentation process has been frequently invoked to explain the observation of maximum size that an aggregate can take before breaking up [19]. Efforts on modeling this process within the context of phytoplankton coagulation has have been minor and the effects of such process on the dynamics of aggregates has not been fully understood.

Obtaining experimental values for parameters that govern the aggregation process, such as, sticking efficiency contact efficiency and growth rate of aggregates has proven to be difficult. Alldredge and Mcgillivary [8] observed that flocculated diatoms in the size range from 0.2 to $7.6 \mathrm{~mm}$ had probability up to 0.88 of sticking to each other. The paper [16] discussed a method for estimating the sticking efficiency from experimental data and a modification of the model of Jackson [14]. Their conclusion was that for some species of phytoplankton stickiness increases when nutrients are depleted and for other species stickiness remained almost constant. In [4] an inverse methodology based on least squares, was used together with an aggregation model developed in [3], in which growth occur only in single cells and not aggregates, to fit data obtained from [13]. Their conclusions were that the modeled contact efficiencies are small, in comparison with the actual ones, giving rise to low densities for large aggregates $\left(>0.6 \mathrm{~mm}^{3}\right)$, a corroboration of 
Hill's [12] observation. In [1] it was suggested that another solution can be the inclusion of growth term for aggregates as opposed to single cells, as has been the case for most modeling efforts so far. Discussion on the difficulty of modeling growth for aggregates and the importance of developing a method for estimating this functions is available in [1].

For a better understanding of the effects of coagulation on the dynamics of phytoplankton we incorporate fragmentation process in a model developed by Ackleh and Fitzpatrick [1]. The resulting model has the following form:

$$
\frac{\partial u}{\partial t}+\frac{\partial(g u)}{\partial x}=F(u)
$$

with the following boundary and initial conditions:

$$
\left\{\begin{array}{l}
g\left(x_{0}\right) u\left(t, x_{0}\right)=K(u) \\
u(0, x)=u_{0}(x)
\end{array}\right.
$$

where

$$
\begin{aligned}
F(u)=\frac{1}{2} & \int_{x_{0}}^{x-x_{0}} \beta(x-y, y) u(t, x-y) u(t, y) d y \\
& -u(t, x) \int_{x_{0}}^{x_{1}} \beta(x, y) u(t, y) d y \\
& -\frac{1}{2} \int_{x_{0}}^{x-x_{0}} \gamma(x-y, y) u(t, x) d y \\
& +\int_{x_{0}}^{x_{1}-x} \gamma(x, y) u(t, x+y) d y-\frac{w u}{Z},
\end{aligned}
$$

and

$$
K(u)=\int_{x_{0}}^{x_{1}} k(x) u(t, x) d x
$$

Here the $x$ variable represents the size $\left(\mathrm{mm}^{3}\right)$ of the aggregate, and the $t$ variable represents time. The function $u(t, x)$ is the density of aggregates of size $x$ 
at time $t$ while the function $g=g(x)$ is the size-dependent growth function. The function $w=w(x)$ is the size-dependent sinking rate and $Z$ is the depth of the surface layer. The term $\frac{w u}{Z}$ in the function $F(u)$ represents the loss of particles of size $x$ due to sinking out of the surface layer. The function $\beta(x, y)$ represents the rate at which an aggregate of size $x$ coalesce with aggregate of size $y$ and similarly the function $\gamma(x, y)$ represents the rate at which particles of sizes $x+y$ fragment to form particles of sizes $x$ and $y$. The first term in $F(u)$ expresses the rate at which collisions occur to form new particles with the size $x$ and $x+d x$ while the second term expresses the rate at which collisions cause particles to be lost from the same interval. The third term describes the loss of particles of size $x$ due to fragmentation to smaller sizes and the last term gives the increase in particles of size $x$ owing to such fragmentation. That the size of any individual aggregate is less than $x_{1}$, the largest observed aggregate $(<10 \mathrm{~mm}$ in diameter $)$, will follow from the assumptions on $g$ and $\beta$.

The main goal of this paper is developing methodology for the determination of the functions $g, \beta, \gamma$ and $k$ from pointwise observations $z_{i j}$ which correspond to the density of aggregates of size $x_{i}$ at time $t_{j}$. Such observation are available in literature (see, e.g., $[13,21]$ ). To determine parameters based on these observations, we define the least square cost functional

$$
J(\beta, \gamma, g, k)=\sum_{i, j}\left|u\left(t_{i}, x_{j} ; \beta, \gamma, g, k\right)-z_{i, j}\right|^{2}
$$

which is minimized over some set $\mathcal{B} \times \mathcal{E} \times \mathcal{G} \times \mathcal{K}$ to be chosen later. In general there are two main difficulties involved in trying to implement this approach: the function $u$ must be computed numerically and the infinite dimensional parameter 
set must be approximated by a finite dimensional set. Thus, for computational purposes, we seek to minimize

$$
J^{N}(\beta, \gamma, g, k)=\sum_{i, j}\left|u^{N}\left(t_{i}, x_{j} ; \beta, \gamma, g, k\right)-z_{i, j}\right|^{2}
$$

over a set $\mathcal{B}^{M} \times \mathcal{E}^{M} \times \mathcal{G}^{M} \times \mathcal{Q}^{M}$. The abstract least square theory (see, [10], page 144) lays out general conditions that must be satisfied in order to guarantee the convergence of minimizers $J^{N}$ to minimizers of $J$. The convergence results we present in this paper allow the application of this abstract least square theory.

This paper is organized as follows. In Section 2 we discuss a the numerical method developed in [1], used for approximating the state equation and prove convergence of this method on $L^{1}\left(x_{0}, x_{1}\right)$. The convergence of parameter approximation is the topic of Section 3. Numerical results are discussed in Section 4. We give some concluding remarks and future studies in Section 5. For completeness we discuss existence and uniqueness of solutions in the Appendix.

\section{Approximation Scheme of The State Equation}

For our analysis we shall use the the state space $L^{1}\left(x_{0}, x_{1}\right)$ with a norm denoted by $\|\cdot\|$. This space is a natural space for this problem since the total population of aggregates at time $t$ is represented by

$$
P(t)=\int_{x_{0}}^{x_{1}} u(x, t) d x, \quad t \geq 0
$$

for meaningful size distributions $u(t, x) \geq 0$, which is just the $L^{1}$-norm of $u(t, \cdot)$. 
We consider the following abstract form of equation (1.1)

$$
\dot{u}=A u+F(u)
$$

where the linear operator $A: \operatorname{dom} A \subset L^{1}\left(x_{0}, x_{1}\right) \rightarrow L^{1}\left(x_{0}, x_{1}\right)$ given by

$$
A \phi=-\frac{\partial}{\partial x}(g \phi)
$$

with

$$
\begin{aligned}
& \operatorname{dom} A=\left\{\phi \in L^{1} \mid g \phi\right. \text { is absolutely continuous, } \\
& \left.\qquad \lim _{x \rightarrow x_{1}}(g \phi)(x)=0,(g \phi)\left(x_{0}\right)=K(\phi)\right\}
\end{aligned}
$$

As can be seen in the Appendix, Lemma A.1 and Theorem A.1. can be established using similar techniques used in [1].

Lemma A.1. For $u_{0} \in L^{1}\left(x_{0}, x_{1}\right)$, (1.1) has a unique mild solution $u(t, x)$ on $[0, T)$ for some $T>0$.

Theorem A.1. If $u_{0} \in \operatorname{dom} A$, then the unique mild solution is a classical one.

In the process of obtaining these results, some important inequalities that indicate the dependence of $F$ on $u$ and the parameters $\beta$ and $\gamma$. In particular, we have

$$
\begin{aligned}
& \|F(u, \beta, \gamma)-F(u, \hat{\beta}, \hat{\gamma})\| \leq \frac{3}{2}\left(\|\beta-\hat{\beta}\|_{\infty}\|u\|^{2}+\|\gamma-\hat{\gamma}\|_{\infty}\|u\|\right)+\|w\|_{\infty} \frac{\|u\|}{Z} \\
& \|F(u)-F(v)\| \leq \frac{3}{2} B_{1}(\|u\|+\|v\|) \mid u-v\left\|+\frac{3}{2} B_{2}\right\| u-v\left\|+\frac{\|w\|_{\infty}}{Z}\right\| u-v \|
\end{aligned}
$$


The above inequalities show that $F$ is locally Lipschitz in $u$, and $F(u, \beta, \gamma)$ is continuous in $\beta$ and $\gamma$. These properties of $F$ will be of importance in the convergence arguments presented in this section and section 3 .

For approximating equation (1.1) we have used a method developed in [1]. We make the following assumptions on our parameters to establish convergence of the method:

(H1) $g$ is continuously differentiable on $\left[x_{0}, x_{1}\right]$.

(H2) $w$ is continuous on $\left[x_{0}, x_{1}\right]$.

(H3) $k$ is continuous on $\left[x_{0}, x_{1}\right]$.

(H4) $\beta$ is continuous on $\left[x_{0}, x_{1}\right] \times\left[x_{0}, x_{1}\right], \beta(x, y)=\beta(y, x)$ and

$$
\left\{\begin{array}{l}
\beta(x, y) \geq 0 \text { if } x+y \leq x_{1} \\
\beta(x, y)=0 \text { if } x+y>x_{1}
\end{array}\right.
$$

(H5) $\gamma$ is continuous on $\left[x_{0}, x_{1}\right] \times\left[x_{0}, x_{1}\right], \gamma \geq 0$ and $\gamma(x, y)=\gamma(y, x)$.

For $N=1,2, \cdots$ and $j=0, \cdots, N-1$ let $x_{j}=x_{0}+\frac{j\left(x_{1}-x_{0}\right)}{N}$ and define

$$
\phi_{j}^{N}=\left\{\begin{array}{l}
1 \text { for } x_{j} \leq x<x_{j+1} \\
0 \text { elsewhere }
\end{array}\right.
$$

For each $N$, these functions form a basis for the subspaces

$$
X^{N}=\left\{f \in L^{1} \mid f=\sum_{j=0}^{N-1} f_{j} \phi_{j}^{N}, f_{j} \in R\right\}
$$

with a norm defined by

$$
\|f\|=\frac{1}{N} \sum_{j=0}^{N-1}\left|f_{j}\right|
$$

Define the projections $\pi^{N}: L^{1} \rightarrow X^{N}$ by 


$$
\pi^{N} f=\sum_{j=0}^{N-1} f_{j} \phi_{j}^{N} \text { with } f_{j}=N \int_{x_{j}}^{x_{j+1}} f(x) d x, \quad j=0, \cdots, N-1
$$

It is well known (e.g., see [9]) that $\pi^{N}$ is the projection of $L^{1}$ onto $X^{N}$ with a norm $\left|\pi^{N}\right|=1$.

We define the approximating generators $A^{N}: X^{N} \rightarrow X^{N}$ of the infinitesimal generator $A$ by:

$$
\left(A^{N} f\right)(x)=-\sum_{j=1}^{N-1} N\left(g\left(x_{j+1}\right) f_{j}-g\left(x_{j}\right) f_{j-1}\right) \phi_{j}^{N}(x)+\sum_{j=0}^{N-1} q\left(x_{j+1}\right) f_{j} \phi_{0}^{N}(x),
$$

for $f \in X^{N}$.

Applying $A^{N}$ to the basis elements we get:

$$
A^{N} \phi_{k}^{N}=-N g\left(x_{k+1}\right) \phi_{k}^{N}+N g\left(x_{k+1}\right) \phi_{k+1}^{N}+q\left(x_{k+1}\right) \phi_{0}^{N}
$$

Hence the matrix representation $\left[A^{N}\right]$ of $A^{N}$ with respect to our basis is given by

$$
\left(\begin{array}{ccccc}
q\left(x_{1}\right)-N g\left(x_{1}\right) & q\left(x_{2}\right) & q\left(x_{3}\right) & \ldots & q\left(x_{N}\right) \\
N g\left(x_{1}\right) & -N g\left(x_{2}\right) & 0 & \ldots & 0 \\
0 & N g\left(x_{2}\right) & -N g\left(x_{3}\right) & \ldots & 0 \\
\ldots \ldots \ldots \ldots \ldots \ldots \ldots \ldots \ldots \ldots \ldots \ldots \ldots \ldots \ldots \ldots \ldots \ldots \ldots \ldots \ldots \ldots & \ldots \ldots \ldots
\end{array}\right)
$$

Thus, as in [1] we can state our approximating problem of (1.1) in following simple operator form:

$$
\left\{\begin{array}{l}
\frac{\partial u^{N}}{\partial t}=A^{N} u^{N}+\pi^{N} F\left(u^{N}\right) \\
u^{N}(0, x)=\pi^{N} u_{0}(x)
\end{array}\right.
$$


To prove convergence of the above approximation, we take the standard Trotter Kato approach (which involves showing stability and consistency of the approximating semigroups,e.g., Theorem 114 page 40 in [10]). To prove stability of scheme we let $T^{N}(t)$ be the semigroup defined on $X^{N}$ with an infinitesimal generator $A^{N}$ (i.e, $\left.T^{N}(t)=e^{A^{N} t}\right)$ then we have

Lemma 2.1. $\left\|T^{N}(t)\right\| \leq e^{\|k\|_{\infty} t}$, for all $N$

Proof. First notice that for $f \in X^{N}$ we have that:

$$
\|f\|=\|f\|_{N}
$$

Hence $X^{N}$ as a subspace of $L^{1}$ is isometrically isomorphic to $R^{N}$. Corresponding to the norm $\|\cdot\|_{N}$ is the usual one matrix norm denoted by $\|B\|_{1}$ for $B \in R^{N \times N}$.

Is is well-known that $\left\|e^{B t}\right\| \leq e^{m(B) t}$, for $t \geq 0$, where

$$
m(B)=\lim _{h \rightarrow 0}\left(\|I+h B\|_{1}-1\right)
$$

In $[9]$ it was shown that

$$
m(B)=\max _{k=0, \cdots, N-1} a_{k}(B), \quad \text { where } \quad a_{k}(B)=B_{k k}+\sum_{i=0, i \neq 1}^{N-1}\left|a_{i k}\right|
$$

Hence for $K=0, \cdots, N-1$ we have that

$$
a_{k}\left(\left[A^{N}\right]\right) \leq\|k\|_{\infty}
$$

Therefore we have that following bound holds

$$
\left\|e^{A^{N} t}\right\|_{1} \leq e^{\|k\|_{\infty} t}
$$


Next we will show that the approximating generators converge on a sufficiently "large subset" of $L^{1}$. For this we need the following lemmas:

Lemma 2.2. The set $D=\left\{f \mid f \in \operatorname{dom} A, \quad f \in C^{1}\left[x_{0}, x_{1}\right]\right\}$ is dense in $L^{1}\left(x_{0}, x_{1}\right)$.

Proof. Define $S=\left\{f \quad \mid f \in C^{1}\left[x_{0}, x_{1}\right] \quad \operatorname{supp} f \subset[0,1)\right\}$. Since it is clear that $S$ is dense in $L^{1}\left(x_{0}, x_{1}\right)$, the result then follows from the proof of Lemma 3.2 in [1], where it was shown that $D$ is dense in $S$.

Lemma 2.3. There exist a $\lambda_{0}$ with $\operatorname{Re} \lambda_{0}>w$ such that $\overline{\left(\lambda_{0}-A\right) D}=L^{1}\left(x_{0}, x_{1}\right)$. Proof. In the proof of Lemma 3.3 in [1] it was shown that $S \subset(\lambda-A) D$, where $S$ is as defined above. Hence the above result is established since $S$ is dense in $L^{1}\left(x_{0}, x_{1}\right)$.

Lemma 2.4. For each $y \in D,\left\|A^{N} \pi^{N} y-\pi^{N} A y\right\|_{N} \rightarrow 0$ as $N \rightarrow \infty$

Proof. As in the proof of Lemma 3.4 in [1] we first note that

$$
\pi^{N} A y=\sum_{j=0}^{N-1}\left(N \int_{x_{j}}^{x_{j+1}}-(g y)^{\prime} d x \phi_{j}^{N}=-\sum_{j=0}^{N-1} N\left(g\left(x_{j+1}\right) y_{j+1}-g\left(x_{j}\right) y_{j}\right) \phi_{j}^{N}\right.
$$

and

$$
A^{N}\left(\pi^{N} y\right)=A^{N}\left(\sum_{j=0}^{N-1}\left(N \int_{x_{j}}^{x_{j+1}} y(\xi) d \xi\right) \phi_{j}^{N}\right)=\sum_{j=0}^{N-1} \alpha_{j} A^{N} \phi_{j}^{N}
$$

with

$$
\alpha_{j}=N \int_{x_{j}}^{x_{j+1}} y(\xi) d \xi
$$


Hence,

$$
\begin{aligned}
\left\|\pi^{N} A y-A^{N} \pi^{N} y\right\|_{N}= & \frac{1}{N} \sum_{j=0}^{N-1}\left|\left(\pi^{N} A y\right)_{j}-\left(A^{N} \pi^{N} y\right)_{j}\right| \\
= & \frac{1}{N}\left(\left|-N g\left(x_{1}\right) y_{1}+N g\left(x_{0}\right) y_{0}+N \alpha_{0} g\left(x_{1}\right)-\sum_{j=0}^{N-1} \alpha_{j} q\left(x_{j+1}\right)\right|\right. \\
& +\sum_{i=1}^{N-1} \mid-N g\left(x_{j+1}\right) y_{j+1}+N g\left(x_{j}\right) y_{j} \\
& \left.+N \int_{x_{j}}^{x_{j+1}} y(x) d x g\left(x_{j+1}\right)-N \int_{x_{j-1}}^{x_{j}} y(x) d x g\left(x_{j}\right) \mid\right)
\end{aligned}
$$

Using Taylor's Theorem we have that the right hand side ( $R H S$ ) satisfies:

$$
\begin{aligned}
R H S \leq & \frac{1}{N}\left(\left|N g\left(x_{0}\right) y_{0}-\sum_{j=0}^{N-1} \alpha_{j} q\left(x_{j+1}\right)\right|\right. \\
& +\left|N g\left(x_{1}\right) y_{1}+N\left(y_{1}-\frac{1}{2 N} y^{\prime}(\hat{\xi})\right) g\left(x_{1}\right)\right| \\
& +\sum_{j=0}^{N-1} \mid-N g\left(x_{j+1}\right) y_{j+1}+N g\left(x_{j}\right) y_{j} \\
& \left.+N\left(y_{j+1}-\frac{1}{2 N} y^{\prime}(\xi)\right) g\left(x_{j+1}\right)-N\left(y_{j}-\frac{1}{2 N} y^{\prime}(\bar{\xi})\right) g\left(x_{j}\right) \mid\right)
\end{aligned}
$$

Where $x_{0}<\hat{\xi}<x_{1}, x_{k-1}<\bar{\xi}<x_{k}$ and $x_{k}<\xi<x_{k+1}$. Simplifying one more step we see that the $R H S$ satisfies

$$
\begin{aligned}
R H S \leq & \frac{1}{N}\left(\left|N g\left(x_{0}\right) y_{0}-\sum_{j=0}^{N-1} \alpha_{j} q\left(x_{j+1}\right)\right|+\frac{1}{2}\left|y^{\prime}(\hat{\xi}) g\left(x_{1}\right)\right|\right. \\
& +\sum_{j=1}^{N-1}\left\{\| y^{\prime}|| \mid g\left(x_{j+1}-g\left(x_{j}\right)\left|+\|g\|_{\infty}\right| y^{\prime}(\xi)-y^{\prime}(\bar{\xi}) \mid\right\}\right.
\end{aligned}
$$

Hence as $N \rightarrow \infty$ then the right hand side $\rightarrow 0$ by the uniform continuity of $y^{\prime}$ and $g$.

Theorem 2.1. For and $t_{0} \in[0, T)$ and any $\phi \in L^{1},\left\|T^{N}(t) \pi^{N} \phi-\pi^{N} T(t) \phi\right\| \rightarrow 0$ uniformly in $t \in\left[0, t_{0}\right]$. 
Proof. The proof follows from the above Lemmas and the Trotter Kato Theorem.

Theorem 2.2. For any $t_{0} \in[0, T]$, the semi-discrete solutions of equation (2.3) converges in $\|\cdot\|$ to the unique solution of (1.1), uniformly on the interval $\left[0, t_{0}\right]$

Proof. Note that the solution of the semidiscrete equation (3.1) satisfies

$$
u^{N}(t)=T^{N}(t) \pi^{N} u_{0}+\int_{0}^{t} T^{N}(t-s) \pi^{N} F\left(u^{N}(s)\right) d s .
$$

Subtracting this expression from the original mild equation and adding and subtracting some terms we arrive at:

$$
\begin{aligned}
\left\|u^{N}(t)-u(t)\right\| \leq & \left\|T^{N}(t) \pi^{N} u_{0}-T(t) u_{0}\right\| \\
& \quad+\int_{0}^{t}\left\|T^{N}(t-s) \pi^{N} F\left(u^{N}(s)\right)-T^{N}(t-s) \pi^{N} F(u(s))\right\| d s \\
& \quad+\int_{0}^{t}\left\|T^{N}(t-s) \pi^{N} F(u(s))-T(t-s) F(u(s))\right\| d s \\
\leq \quad & T^{N}(t) \pi^{N} u_{0}-T(t) u_{0}\left\|+L_{1} \int_{0}^{t}\right\| u^{N}(s)-u(s) \| d s \\
& \quad+\int_{0}^{t}\left\|T^{N}(t-s) \pi^{N} F(u(s))-T(t-s) F(u(s))\right\| d s .
\end{aligned}
$$

Where $L_{1}$ is a constant that depends on the bounds for $T^{N}(\cdot), \pi^{N}$ and the local Lipschitz property of $F$ given in (2.2) ( in using the local Liphscitz property it is important to note that the approximate solutions are uniformly bounded in $N$ due to the uniform bound of the approximate semigroups). Hence we see that an application of Theorem 2.1. and Gronwall's inequality will establish the result. 


\section{The Least Squares Problem}

To begin our parameter estimation problem, we will define the sets of parameter over which we will minimize our functional $J$ and $J^{N}$. Let

$$
\widetilde{Q}=L^{\infty}\left[\left(x_{0}, x_{1}\right) \times\left(x_{0}, x_{1}\right)\right] \times L^{\infty}\left[\left(x_{0}, x_{1}\right) \times\left(x_{0}, x_{1}\right)\right] \times C^{1}\left[x_{0}, x_{1}\right] \times C\left[x_{0}, x_{1}\right]
$$

where $L^{\infty}\left[\left(x_{0}, x_{1}\right) \times\left(x_{0}, x_{1}\right)\right]$ denotes the usual space of essentially bounded mea-

surable functions. We next define a compact subset of $\widetilde{Q}$ in order to obtain a parameter set which fulfill the requirements of the abstract theory.

$$
\begin{aligned}
\mathcal{B}=\left\{\phi \in L^{\infty}\left[\left(x_{0}, x_{1}\right) \times\left(x_{0}, x_{1}\right)\right] \mid\right. & 0 \leq \phi(x, y), \\
\left.\phi(x, y)=0 \text { for } x+y>x_{1},\|\phi\|_{W^{1, \infty}} \leq M_{1}\right\} & \left.\mathcal{E} \leq \phi(x, y),\|\phi\|_{W^{1, \infty}} \leq M_{2}\right\} \\
\mathcal{E}=\left\{\phi \in L^{\infty}\left[\left(x_{0}, x_{1}\right) \times\left(x_{0}, x_{1}\right)\right] \mid 0<g(x), x \in\left[x_{0}, x_{1}\right), g\left(x_{1}\right)=0,\|g\|_{H^{2}} \leq M_{3}\right\} & \left\{g \in C^{1}\left[x_{0}, x_{1}\right] \mid 0 \leq q(x), x \in\left[x_{0}, x_{1}\right],\|q\|_{H^{1}} \leq M_{4}\right\}
\end{aligned}
$$

The set $Q=\mathcal{B} \times \mathcal{E} \times \mathcal{G} \times \mathcal{K}$ is easily seen to be compact in $\widetilde{Q}$, using Sobolev imbeddings and the Arzela Ascoli theorem.

In order to establish the convergence of the parameter approximation we use the abstract theory in [10] (Page 144). The most difficult and important step requires showing that the approximating solutions of equation (2.3) converge to the unique solution of (1.1)-(1.4) and the convergence is uniform in the parameter. As in Section 2 (based on the Trotter Kato theory) we have to show that the approximating generators $A^{N}$ converge on the subset $D$ defined in Lemma 2.2 to 
the operator $A$, and the convergence is uniform in the parameters $q=(\beta, \gamma, g, k)$.

Lemma 3.4 For each $y \in D,\left\|A^{N}(q) \pi^{N} y-\pi^{N} A(q) y\right\|_{N} \rightarrow 0$ as $N \rightarrow \infty$ uniformly in $q$.

Proof. As in [10] page 144 we need to show that for any sequence $q^{N} \rightarrow q$ in $Q$ we have $\left\|A^{N}\left(q^{N}\right) \pi^{N} y-\pi^{N} A(q) y\right\|_{N} \rightarrow 0$ as $N \rightarrow \infty$. From Lemma 2.4 we need only to show that $\left\|A^{N}\left(q^{N}\right) \pi^{N} y-A^{N}(q) \pi^{N} y\right\| \rightarrow 0$.

Using the definition of the operator $A^{N}$ we have:

$$
\begin{aligned}
& \left\|A^{N}\left(q^{N}\right) \pi^{N} y-A^{N}(q) \pi^{N} y\right\|_{N}=\frac{1}{N} \sum_{j=0}^{N-1} \mid\left(\left(A^{N}\left(q^{N}\right) \pi^{N} y\right)_{j}-\left(A(q) \pi^{N} y\right)_{j} \mid\right. \\
& =\frac{1}{N}\left(\left|\sum_{j=0}^{N-1} \alpha_{j}\left(k^{N}\left(x_{j}\right)-k\left(x_{j}\right)\right)+\alpha_{0} N\left(g^{N}\left(x_{1}\right)-g\left(x_{1}\right)\right)\right|\right. \\
& +\sum_{j=1}^{N-1} \mid N \alpha_{j} g^{N}\left(x_{j+1}\right)-N \alpha_{j-1} g^{N}\left(x_{j}\right)-N \alpha_{j} g\left(x_{j+1}+N \alpha_{j-1} g\left(x_{j}\right) \mid\right.
\end{aligned}
$$

Adding and subtracting terms and using the definition of $\alpha_{j}$ we see that the right hand side (RHS) satisfies:

$$
\begin{aligned}
\text { RHS } \leq & \|y\|_{\infty}\left(\left\|k^{N}-k\right\|_{\infty}+\left\|g^{N}-g\right\|_{\infty}\right) \\
& +\frac{1}{N} \sum_{j=1}^{N-1} \mid N^{2} \int_{x_{j}}^{x_{j+1}} y d x\left(g^{N}\left(x_{j+1}\right)-g^{N}\left(x_{j}\right)\right) \\
& +g^{N}\left(x_{j}\right)\left(N^{2} \int_{x_{j}}^{x_{j+1}} y d x-N^{2} \int_{x_{j-1}}^{x_{j}} y d x\right) \\
& -N^{2} \int_{x_{j}}^{x_{j+1}} y d x\left(g\left(x_{j+1}-g\left(x_{j}\right)\right)\right. \\
& -g\left(x_{j}\right)\left(N^{2} \int_{x_{j}}^{x_{j+1}} y d x-N^{2} \int_{x_{j-1}}^{x_{j}} y d x\right) \mid
\end{aligned}
$$

Using Taylor's theorem we have:

$$
R H S \leq\|y\|_{\infty}\left(\left\|k^{N}-k\right\|_{\infty}+\left\|g^{N}-g\right\|_{\infty}\right)
$$




$$
\begin{aligned}
& +\frac{1}{N} \sum_{j=1}^{N-1} \mid N \int_{x_{j}}^{x_{j+1}} y d x N\left[\left(g^{N}\left(x_{j+1}\right)-g^{N}\left(x_{j}\right)\right)-\left(g\left(x_{j+1}-g\left(x_{j}\right)\right)\right]\right. \\
& +g^{N}\left(x_{j}\right) N\left(y\left(x_{j+1}\right)-\frac{1}{2 N} y^{\prime}\left(\xi_{1}\right)-y\left(x_{j}\right)+\frac{1}{2 N} y^{\prime}\left(\xi_{2}\right)\right) \\
& +g\left(x_{j}\right) N\left(y\left(x_{j+1}\right)-\frac{1}{2 N} y^{\prime}\left(\xi_{3}\right)-y\left(x_{j}\right)+\frac{1}{2 N} y^{\prime}\left(\xi_{4}\right)\right) \mid
\end{aligned}
$$

Where $x_{j}<\xi_{1}<x_{j+1}$ and $x_{j-1}<\xi_{2}<x_{j}$ (similarly for $\xi_{3}, \xi_{4}$ ). Hence, since $g^{N} \rightarrow g$ in $C^{1}\left[x_{0}, x_{1}\right]$ and $q^{N} \rightarrow q$ in $C\left[x_{0}, x_{1}\right]$ we have that $R H S \rightarrow 0$ as $N \rightarrow \infty$.

Theorem 3.1. For any $t_{0} \in[0, T),\left\|T^{N}(q, t) \pi^{N} \phi-\pi^{N} T(q, t) \phi\right\| \rightarrow 0$ uniformly in $t \in\left[0, t_{0}\right]$ and $q \in Q$.

Proof. is a consequence of the Trotter Kato theorem and Lemma 3.4.

Theorem 3.2 Suppose that $q^{N} \rightarrow q$ in $Q$ then we have that for any $t_{0} \in[0, T)$, $u^{N}\left(t, q^{N}\right) \rightarrow u(t, q)$ uniformly on $\left[0, t_{0}\right]$, in $L^{1}$.

Proof. We subtract the mild solution of $u^{N}\left(t, q^{N}\right)$ from that of $u(t, q)$ to get:

$$
\begin{aligned}
& \left\|u^{N}\left(t, q^{N}\right)-u(t, q)\right\| \leq\left\|T^{N}\left(t, q^{N}\right) \pi^{N} u_{0}\left(q^{N}\right)-T(t, q) u_{0}(q)\right\| \\
& \quad+\int_{0}^{t}\left\|T^{N}\left(t-s, q^{N}\right) \pi^{N} F\left(s, u^{N} ; q^{N}\right)-T(t-s, q) F(s, u ; q)\right\| d s .
\end{aligned}
$$

let us consider first the second term. Setting $T^{N}\left(t, q^{N}\right)=T^{N}(t)$ we have

Suppose that $t \in\left[0, t_{0}\right]$, then

$$
\begin{aligned}
& \int_{0}^{t}\left\|T^{N}(t-s) \pi^{N} F\left(s, u^{N} ; q^{N}\right)-T(t-s) F(s, u ; q)\right\| d s \\
& \leq \int_{0}^{t}\left\|T^{N}(t-s) \pi^{N}\left(F\left(s, u^{N} ; q^{N}\right)-F\left(s, u ; q^{N}\right)\right)\right\| d s \\
& +\int_{0}^{t} \mid T^{N}(t-s) \pi^{N}\left(F\left(s, u ; q^{N}\right)-F(s, u ; q)\right) \| d s
\end{aligned}
$$




$$
\begin{aligned}
& +\int_{0}^{t}\left\|T^{N}(t-s) \pi^{N} F(s, u ; q)-T(t-s) F\left(s, u ; q^{N}\right)\right\| d s \\
& \leq K_{1} \int_{0}^{t}\left\|u^{N}(s)-u(s)\right\| d s \\
& +K_{2} \int_{0}^{t}\left\|F\left(s, u ; q^{N}\right)-F(s, u ; q)\right\| d s \\
& +\int_{0}^{t} T^{N}(t-s) \pi^{N} F(s, u ; q)-T(t-s) F(s, u ; q) \mid d s,
\end{aligned}
$$

where $K_{1}, K_{2}$ depend on the bounds for $\left\{\pi^{N}\right\}_{N=1}^{\infty}, T(\cdot)$, and $K_{2}$ depends on the local Lipschitz constant for $F$ given in (2.2). We note that the uniform boundedness of the solutions, which can easily be established due to the uniform boundedness of the semigroups is essential for using the local Lipschitz property of the function $F$.

Hence we have that

$$
\begin{aligned}
\left\|u^{N}(t)-u(t)\right\| \leq \| & T^{N}\left(t, q^{N}\right) \pi^{N} u_{0}\left(q^{N}\right)-T(t, q) u_{0}(q) \| \\
& +K_{2} \int_{0}^{t}\left\|F\left(s, u ; q^{N}\right)-F(s, u ; q)\right\| d s \\
& +\int_{0}^{t} T^{N}(t-s) \pi^{N} F(s, u ; q)-T(t-s) F(s, u ; q) \mid d s \\
& +K_{3} \int_{0}^{t}\left|u^{N}(s)-u(s)\right| d s, \\
\leq & H^{N}(t)+K_{3} \int_{0}^{t}\left\|u^{N}(s)-u(s)\right\| d s,
\end{aligned}
$$

using Theorem 3.1 and the continuity of $F$ with respect to the parameters, we have that $H^{N}(t) \rightarrow 0$ as $N \rightarrow \infty$. Hence a straightforward application of Gronwall's inequality will establish the result.

\section{Numerical Results}

In this section we present some numerical simulations. In the first example 
we illustrate the effects of fragmentation in the model. In the second and third examples we give some basic computations that explore the accuracy of the inverse method. In all of the simulation below we have used $x_{0}=0, x_{1}=1$, a first order discretization in time with $\Delta t=0.004$ and a final time $t=1$. For the size discretization we chose $N=50$ (i.e, $\Delta x=0.02$ ). For the parameters $g, k, w, \beta$ and $\gamma$ we used the following choice of functions:

$$
\begin{gathered}
k(x)=0.3 * x, \quad g(x)=(1-x) *(x+0.2), \quad w=0 ; \\
\beta(x, y)=\left\{\begin{array}{cl}
10 *(x+y)^{2 / 3} *\left(1-(x+y)^{2 / 3}\right) & \text { if } x+y \leq 1 \\
0 & \text { if } x+y>1
\end{array}\right. \\
\gamma(x, y)=\left\{\begin{array}{cl}
\exp \left(\frac{1}{(2(x+y)-1)^{2}-1}\right) & \text { if } x+y \leq 1 \\
0 & \text { if } x+y>1
\end{array}\right.
\end{gathered}
$$

For the initial distribution function we chose

$$
u_{0}(x)=\left\{\begin{array}{ccc}
4 * \exp \left(\frac{1}{\mid 4 * x-1)\left.\right|^{2}-1}\right) & \text { if } & x \leq \frac{1}{2} \\
0 & \text { if } & x>\frac{1}{2}
\end{array}\right.
$$

1) Effect of fragmentation on the dynamics of phytoplankton.

We have simulated our system of equations (1.1)-(1.4) with and without the fragmentation terms and presented the distributions at the final time in Fig. 1. The results show that fragmentation process tends to shift the distribution of aggregates to the left by breaking the large aggregates into smaller ones and hence resulting in high densities for smaller aggregate sizes. 


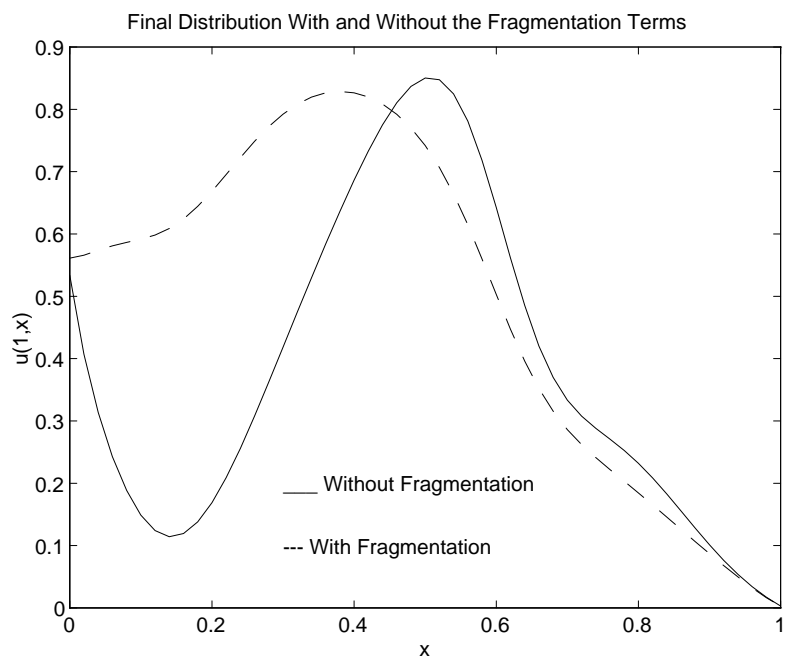

Fig 1. This graph represents the distribution of the aggregates at the final time $t=1$ when fragmentation process is included versus the final distribution when the fragmentation process is not included (i.e, $\gamma=0$ ).

\section{2) Parameter Estimation}

To illustrate the computational methods analyzed herein, we estimated the parameters $k, g, \beta$ and $\gamma$ from computationally generated data using the numerical method discussed in Section 2 and the above parameters.

For the estimation method we have assumed that

$$
\begin{gathered}
k(x)=c_{1} * x, \quad g(x)=c_{2}\left(c_{3}+x\right)(1-x) \\
\beta(x, y)= \begin{cases}c_{4} *(x+y)^{c_{5}} *\left(c_{6}-(x+y)^{c_{5}}\right. & \text { if } x+y \leq 1 \\
0 & \text { if } x+y>1\end{cases}
\end{gathered}
$$

and estimated the constants $c_{1}, \cdots, c_{6}$. Since very little is known about $\gamma$ we have approximated this function using 10 linear splines with the assumption that 
$\gamma(x, y)=\hat{\gamma}(x+y)$, i.e, $\gamma^{10}(z)=\sum_{i=1}^{10} \alpha_{i} B_{i}^{10}(z)$, where $B_{i}(z)$ is the one dimensional linear spline, modified to satisfy zero boundary values. Such an assumption is not unreasonable since $x_{0}$ is the size of single cell aggregates and hence can't be broken into smaller size aggregate and similarly since $x_{1}$ is the largest aggregate size then the densities are zero there. We have started with the constant function $\gamma=4$ as an initial guess for the nonlinear least square solver LMDIF1 obtained from minpack library in all our computations.

For data, we sampled the densities $u\left(t_{i}, x_{j}\right)\left(t_{i}=i * 0.1, i=1 \cdots 10\right.$, and $\left.x_{j}=\frac{j-1}{15}, j=1 \cdots 15\right)$ on the interval $[0,1] \times[0,1]$, as generated with the above model. To examine the behavior of the least squares identification procedure, we used as data the actual model generated densities, as well as the densities modified by Gaussian noise: $z_{i, j}=u\left(t_{i}, x_{j}\right) *\left(1+\epsilon_{i, j}\right)$, was used for data, with $\epsilon_{i, j}$ a random sample from a zero mean Gaussian random number generator. We used $\sigma=.01$ and $\sigma=0.03$ for standard deviations for the noise.

In order to implement the above compactness constraints we used the regularized least squares cost functional of the form

$$
J(\beta, \gamma, g, k)=\sum_{i, j}\left|u\left(t_{i}, x_{j} ; \beta, \gamma, g, k\right)-z_{i, j}\right|^{2}+c \int_{0}^{1}\left|\frac{d}{d z} \gamma(z)\right|^{2} d z
$$

The parameter $c$ varied from $10^{-6}$ to $10^{-4}$ depending on the noise level.

In the case where no noise is added to our model output we present in Table 1, the estimated versus the true $c_{1}, \cdots, c_{6}$ constants, and in Figure 2 the estimated $\gamma$ versus the true function $\gamma$ is presented. 


\begin{tabular}{|c|l|l|l|}
\hline \multicolumn{4}{|c|}{ Table 1 } \\
\hline \hline \multicolumn{3}{|c|}{ No Added Noise } \\
\hline \hline param. & Initial Value & Estimated Value & True Value \\
\hline$c_{1}$ & 0.2 & 0.2994 & 0.3 \\
$c_{2}$ & 0.2 & 0.5006 & 0.5 \\
$c_{3}$ & 0.1 & 0.1993 & 0.2 \\
$c_{4}$ & 8.0 & 9.9941 & 10.0 \\
$c_{5}$ & 1 & 0.6558 & $\frac{2}{3}$ \\
$c_{6}$ & 1.2 & 1.0051 & 1 \\
\hline
\end{tabular}

Table 1. The estimated versus the true $c_{1}, \cdots, c_{6}$ parameters.

In Table 2, we present the estimated versus the true $c_{1}, \cdots, c_{6}$ parameters when $\sigma=.01$, and Figure 2 presents the estimated versus the true function $\gamma(x+y)$ using 10 linear splines. We then use $\sigma=0.03$ and present the estimated parameters in Table 3 and Figure 3.

\begin{tabular}{|c|l|l|l|}
\hline \multicolumn{4}{|c|}{ Table 2 } \\
\hline \hline \multicolumn{3}{|c|}{$\sigma=0.01$} \\
\hline \hline param. & Initial Value & Estimated Value & True Value \\
\hline$c_{1}$ & 0.2 & 0.2803 & 0.3 \\
$c_{2}$ & 0.2 & 0.5243 & 0.5 \\
$c_{3}$ & 0.1 & 0.1781 & 0.2 \\
$c_{4}$ & 8.0 & 9.8851 & 10.0 \\
$c_{5}$ & 1 & 0.6732 & $\frac{2}{3}$ \\
$c_{6}$ & 1.2 & 1.0000 & 1 \\
\hline
\end{tabular}

Table 2 . The estimated versus the true $c_{1}, \cdots, c_{6}$ parameters when the standard deviation of the noise is $\sigma=0.01$. 


\begin{tabular}{|c|l|l|l|}
\hline \multicolumn{3}{|c|}{ Table 3 } \\
\hline \hline \multicolumn{3}{|c|}{$\sigma=0.03$} \\
\hline \hline param. & Initial Value & Estimated Value & True Value \\
\hline$c_{1}$ & 0.2 & 0.2438 & 0.3 \\
$c_{2}$ & 0.2 & 0.5741 & 0.5 \\
$c_{3}$ & 0.1 & 0.1406 & 0.2 \\
$c_{4}$ & 8.0 & 9.538 & 10.0 \\
$c_{5}$ & 1 & 0.6938 & $\frac{2}{3}$ \\
$c_{6}$ & 1.2 & 0.9988 & 1 \\
\hline
\end{tabular}

Table 3 . The estimated versus the true $c_{1}, \cdots, c_{6}$ parameters when the standard deviation of the noise is $\sigma=0.03$.

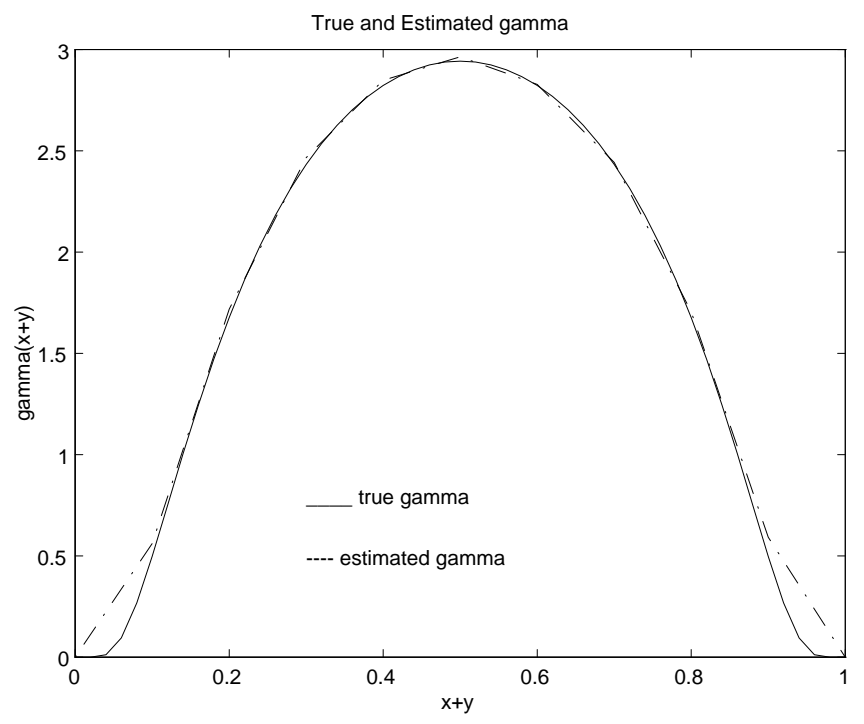

Fig 2. This graph represents the estimated versus the true parameter $\gamma(x+y)$ when no noise is added (the data is the model output). We used 10 linear splines in this estimation. 


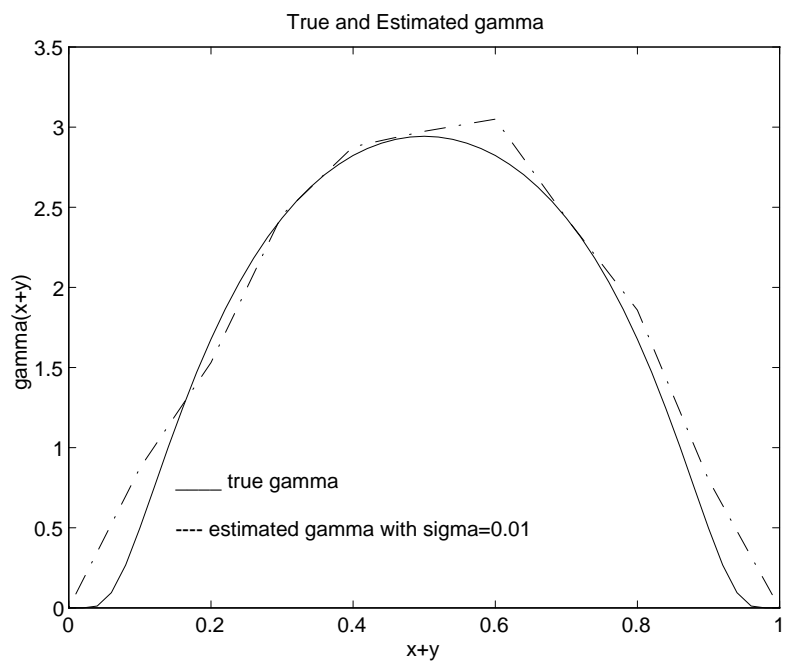

Fig 3. This graph represents the estimated versus the true parameter $\gamma(x+y)$ when the standard deviation of the added noise, $\sigma=0.01$. We used 10 linear splines in this estimation.

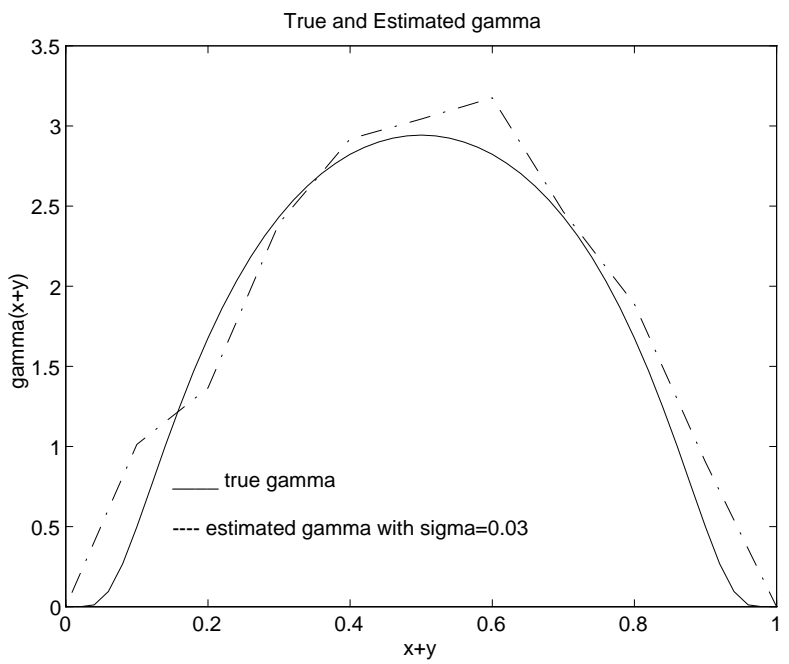

Fig 4. This graph represents the estimated versus the true function $\gamma(x+y)$ when the standard deviation of the added noise $\sigma=0.03$. Again we used 10 linear splines in this estimation. 


\section{Concluding Remarks}

In this paper we have developed a methodology for estimating parameters in structured algal population model with the aggregation process. The initial computational results appear to be very promising. Our future effort will focus on using this methodology in comparing output of similar models such as that developed in [3] to data available in literature [14, 21].

Acknowledgments- The author would like to thank B.G. Fitzpatrick for the many useful comments and discussions regarding the material in this manuscript.

\section{Appendix: Existence, Uniqueness and Regularity}

We take a rather standard semigroup approach for semilinear partial differential equations to establish well posedness of solutions. We require less regularity on our parameters for establishing existence uniqueness then is required by the numerical method. Hence for this section the following assumptions are required to be satisfied by the model parameters in order to establish existence and uniqueness. These conditions have been used in other structured population problems $([9,2])$.

(AH1) $g \in W^{1, \infty}\left(x_{0}, x_{1}\right), g(x)>0$ for $x_{0} \leq x<x_{1}$ and $g\left(x_{1}\right)=0$

(AH2) $w \in L^{\infty}\left(x_{0}, x_{1}\right)$ and $w \geq 0$ a.e on $\left[x_{0}, x_{1}\right]$

(AH3) $q \geq 0$ a.e on $\left[x_{0}, x_{1}\right]$ and $q \in L^{\infty}\left(x_{0}, x_{1}\right)$ 
$(\mathrm{AH} 4) \beta \in L^{\infty}\left(\left[x_{0}, x_{1}\right] \times\left[x_{0}, x_{1}\right]\right), \beta(x, y)=\beta(y, x)$ and

$$
\left\{\begin{array}{l}
\beta(x, y) \geq 0 \text { if } x+y \leq x_{1} \\
\beta(x, y)=0 \text { if } x+y>x_{1}
\end{array}\right.
$$

(AH5) $\gamma \in L^{\infty}\left(\left[x_{0}, x_{1}\right] \times\left[x_{0}, x_{1}\right]\right), \gamma(x, y)=\gamma(y, x)$ and $\gamma \geq 0$.

We have from [9] that $A$ is an infinitesimal generator for a $C_{0}$-semigroup $T(t)$ that satisfies $\|T(t)\| \leq e^{\omega t}$.

Using the semigroup, we consider the mild form of the equation, given by

$$
\rho(t)=T(t) \rho_{0}+\int_{0}^{t} T(t-s) F(\rho(s)) d s,
$$

which provides the first step in solving (1.1).

Lemma 2.1. For $u_{0} \in L^{1}\left(x_{0}, x_{1}\right)$, (1.1) has a unique mild solution $u(t, x)$ on $\left[0, t_{\max }\right)$ for some $t_{\max }>0$.

\section{proof.}

Since $F$ is independent of $t$, we need only to show (Pazy, Theorem 1.4, page 185) that $F: L^{1} \rightarrow L^{1}$ and that $F$ is locally Lipschitz. First we show that $F: L^{1} \rightarrow L^{1}$. From (AH4) and (AH5) we have that $\|\beta\|_{\infty} \leq B_{1}$ and $\|\gamma\|_{\infty} \leq B_{2}$ for some constants $B_{1}, B_{2}$.

We first will show that the first integral term of $F(u)$ is a mapping from $L^{1} \rightarrow L^{1}$ Define $k(x, y)=\chi_{[0, x]}(y) * \beta(x-y, y) * u(x-y)$. Then for $u \in L^{1}$ a simple application of the generalized Young's inequality (e.g, [11], page 13) on the space $\left[x_{0}, x_{1}\right] \times\left[x_{0}, x_{1}\right]$ will give the following estimate on the first term

$$
\frac{1}{2} \int_{x_{0}}^{x_{1}}\left|\int_{x_{0}}^{x-x_{0}} \beta(x-y, y) u(x-y) u(y) d y\right| d x \leq \frac{1}{2} B_{1}\|u\|^{2}
$$


In a similar manner one can find a bound on the second term of $F(u)$. (Establishing bounds for the rest of the terms is a straightforward manner due to the linearity of these terms). Hence this shows that $F: L^{1} \rightarrow L^{1}$ and the following bound holds

$$
\|F(u)\| \leq \frac{3}{2}\left(B_{1}\|u\|^{2}+B_{2}\|u\|\right)+\|w\|_{\infty} \frac{\|u\|}{z} .
$$

To show that $F$ is locally Lipschitz, we let $u, v \in L^{1}$ with $\|u\| \leq C$ and $\|v\| \leq C$ we want to show that

$$
\|F(u)-F(v)\| \leq L(C) \mid u-v \|
$$

Again we start by considering the first term of $F(\rho)$ :

$$
\begin{gathered}
\int_{x_{0}}^{x_{1}}\left|\int_{x_{0}}^{x-x_{0}} \beta(x-y, y)(u(x-y) u(y)-v(x-y) v(y)) d y\right| \\
\leq \int_{x_{0}}^{x-x_{0}} \mid \beta(x-y, y)(u(x-y) u(y)-u(x-y) v(y) \\
\quad+u(x-y) v(y)-v(x-y) v(y)) \mid d y d x \\
\leq B_{1}(\|u\|+\|v\|)\|u-v\| .
\end{gathered}
$$

The last inequality is again an application of generalized Young's inequality.

One can obtain a similar bound for the rest of the terms of $F(\rho)$ and hence we can show that

$$
\begin{gathered}
\|F(u)-F(v)\| \leq \frac{3}{2} B_{1}(\|u\|+\|v\|) \mid u-v\left\|+\frac{3}{2} B_{2}\right\| u-v\left\|+\frac{\|w\|_{\infty}}{Z}\right\| u-v \| \\
=L(C) \mid u-v \|
\end{gathered}
$$


Hence $F(\rho)$ is locally Lipschitz and this establishes the desired result.

Lemma 2.2. If $\rho_{0} \in \operatorname{dom} A$, then the unique mild solution is a classical one.

\section{Proof.}

It is enough to show that $F: L^{1} \rightarrow L^{1}$ is continuously differentiable from $L^{1} \rightarrow L^{1}$ (see Pazy, Theorem 1.5, page 187). Let $u \in L^{1}$ we want to show that

$$
F(u+h)-F(u)=L(u, h)+O(u, h)
$$

for $h \in L^{1}$, where $L(u,.) \in \mathcal{L}\left(L^{1}\right)$, the map $u \rightarrow L(u, \cdot)$ is continuous in the uniform operator topology, and that

$$
\lim _{\|h\| \rightarrow 0} \frac{\|O(u, h)\|}{\|h\|}=0
$$

First we note that

$$
\begin{aligned}
F(u+h)-F(u)= & \frac{1}{2} \int_{x_{0}}^{x-x_{0}} \beta(x-y, y)(h(x-y) u(y)+u(x-y) h(y)) d y \\
& -u(x) \int_{x_{0}}^{x_{1}} \beta(x, y) h(y) d y+h(x) \int_{x_{0}}^{x_{1}} \beta(x, y) u(y) d y- \\
& -\frac{1}{2} \int_{x_{0}}^{x-x_{0}} \gamma(x-y, y) h(y) d y \\
& +\int_{x_{0}}^{x_{1}-x} \gamma(x, y) h(x+y) d y-\frac{w h(x)}{Z} \\
& +\frac{1}{2} \int_{x_{0}}^{x-x_{0}} \beta(x-y, y) h(x-y) h(y) d y \\
& -h(x) \int_{x_{0}}^{x_{1}} \beta(x, y) h(y) d y=L(u, h)+O(u, h) .
\end{aligned}
$$

where 


$$
\begin{aligned}
O(u, h)= & \frac{1}{2} \int_{x_{0}}^{x-x_{0}} \beta(x-y, y) h(x-y) h(y) d y \\
& -h(x) \int_{x_{0}}^{x_{1}} \beta(x, y) h(y) d y
\end{aligned}
$$

For $h \in L^{1}$ we have shown earlier that

$$
\|O(u, h)\| \leq \frac{3}{2} B_{1}\|h\|^{2}
$$

hence it is clear that

$$
\lim _{\|h\| \rightarrow 0} \frac{\|O(u, h)\|}{\|h\|}=0 .
$$

Linearity of the operator $L$ is clear, thus, it is left to show that the map $u \rightarrow L(u, \cdot)$ is continuous in the uniform topology. Let $u_{n} \rightarrow u$ in $L^{1}$. Then

$$
\begin{aligned}
\left\|L\left(u_{n}, h\right)-L(u, h)\right\|= & \int_{x_{0}}^{x_{1}} \mid \frac{1}{2} \int_{x_{0}}^{x-x_{0}} \beta(x-y, y)\left(h(x-y)\left(u_{n}(y)-u(y)\right)\right. \\
& \left.+\left(u_{n}(x-y)-u(x-y)\right) h(y)\right) d y \\
& -\left(u_{n}(x)-u(x)\right) \int_{x_{0}}^{x_{1}} \beta(x, y) h(y) d y \\
& +h(x) \int_{x_{0}}^{x_{1}} \beta(x, y)\left(u_{n}(y)-u(y)\right) d y \mid d x \\
\leq & B_{1}\left\|u_{n}-u\right\|\|h\|+2 B_{1}\left\|u_{n}-u\right\|\|h\|
\end{aligned}
$$

Hence we have

$$
\left\|L\left(u_{n}, h\right)-L(u, h)\right\| \leq 3 B_{1}\left\|u_{n}-u\right\|\|h\|
$$

so that

$$
\| L\left(u_{n}, .-L(u, .)\left\|_{\mathcal{L}\left(L^{1}\right)}=\sup _{\|h\| \leq 1}\right\| L\left(u_{n}, h\right)-L(u, h) \| \rightarrow 0,\right.
$$

which establishes the desired result. 


\section{References}

[1] Ackleh, A. S. and B. G. Fitzpatrick (1994) Modeling aggregation and growth processes in an Algal population model: Analysis and computation. CRSC Technical Report 94-14. Submitted to Journal of Mathematical Biology.

[2] Ackleh, A. S. , B. G. Fitzpatrick and T. G. Hallam (1994) Approximation and parameter estimation for algal aggregation models. Mathematical Models and Methods in Applied Sciences, 4, 291-311.

[3] Ackleh A. S., T. G. Hallam and W. O. Smith (1994) Influences of aggregation and grazing on phytoplankton dynamics and fluxes: an individual-based modeling approach. Nonlinear World, 1, 473-492.

[4] Ackleh A. S., T. G. Hallam and H. C. Muller-Landau (1994) Estimation of sticking and contact efficiencies in aggregation of phytoplankton: The 1993 SIGMA tank experiment. CRSC Technical Report 94-21. Deep Sea Research Journal, a Special Issue on Aggregation (in Press).

[5] Alldredge, A. L. and C. C. Gotschalk (1988a) Direct observations of the mass flocculation of diatom blooms: characteristics, settling velocities and formation of diatom aggregates. Deep-Sea Research, 36, 159-171.

[6] Alldredge, A. L. and C. C. Gotschalk (1988b) In situ settling behavior of marine snow. Limnology and Oceanography, 33, 339-351.

[7] Alldredge, A. L., T.C. Granata, C.C. Gotschalk, T.D. Dickey (1990) The physical strength of marine show and its implications for particle disaggregation in the ocean. Limnology and Oceanography, 35, 1415-1428. 
[8] Alldredge, A. L. and P. Mcgillivary(1991) The attachment probabilities of marine snow and their implications for particle coagulation in the ocean. Deep-Sea Research, 38, 431-443.

[9] Banks H. T.and F. Kappel (1989) Transformation semigroups and $L^{1}$ approximation for size structured population models. Semigroup Forum, 38, $141-155$.

[10] Banks H. T. and K. Kunisch (1989) Estimation techniques for distributed parameter systems. Birkhauser.

[11] Folland, G. B. (1976) Introduction to partial differential equations Princeton University Press, Princeton, New Jersey.

[12] Hill, P. S. (1992) Reconciling aggregation theory with observed vertical fluxes following phytoplankton blooms. Journal of Geophysical Research, 97, 22952308 .

[13] Jackson G. (1994) SIGMA Data Report 1. Santa Barbara Tank Experiment.

[14] Jackson G. (1990) A model of formation of marine algal flocs by physical coagulation processes. Deep-Sea Research, 37, 1197-1211.

[15] Jackson G. and S. Lochmann (1992) Effect of coagulation on nutrient and light limitation of an algal bloom, Limnology and Oceonography, 37, 77-89.

[16] Kiorboe T. , K. P. Anderson and H. G. Dam (1990) Coagulation efficiency and aggregate formation in marine phytoplankton. Marine Biology, 107, 235-245. 
[17] McCave, I. N. (1984) Size-spectra and aggregation of suspended particles in the deep ocean. Deep-Sea Research, 31, 329-352.

[18] O’Mellia, C. R. and K. S. Bowman (1984) Origins and effects of coagulation in lakes. Schweiz. Z. Hydrol. 46 64-85.

[19] Parker, D.S., W.J. Jaufman, and D. Jenkins (1972) Floc Breakup in turbulent flocculation processes. Journal of the Sanitary Engineering Division, Proceedings of the American Society of Civil Engineers, 98 SA1, 79-99.

[20] Pazy A. Semigroup of Linear Operators and Applications to Partial Differential Equations. Springer-Verlag.

[21] Riebesell U. (1991 a,b) Particle aggregation during a diatom bloom. 1. Physical aspects. 2. Biological aspects. Marine Ecology Progress Series, 69, 273-280, 281-291.

[22] Riebesell, U. and D. A. Wolf-Gladrow (1992) The relationship between physical aggregation of phytoplankton and particle flux: a numerical model. DeepSea Research, 39, 1085-1102.

[23] Smetack, V. S. (1985) Role of sinking in diatom like-history cycles, evolutionary and geological significance. Marine Biology, 84, 239-251.

[24] Weilenmann, U., C. R. O’Melia and W. Stumm (1989) Particle transport in lakes: Models and measurements. Limnology and Oceanography, 34 1-18. 